$\xi=\square$

\title{
Structural and piezoelectric coefficients of AlP under pressure
}

\author{
Salah Daoud $^{1}$ *, Rabie Mezouar ${ }^{1}$, Abdelfateh Benmakhlouf ${ }^{2}$ \\ ${ }^{1}$ Laboratory of Materials and Electronic Systems (LMSE), Mohamed Elbachir El Ibrahimi, Bordj Bou Arreridj University, \\ 34000 Bordj Bou Arreridj, Algeria \\ ${ }^{2}$ Department of Mechanical Engineering, Faculty of Technology, University of Amar Telidji-Laghouat, 03000 Laghouat, Algeria \\ *Corresponding author E-mail: salah_daoud07@yahoo.fr
}

\begin{abstract}
The present work aims to investigate the structural parameters and the piezoelectric coefficients of cubic zinc-blende Aluminum phosphide (AlP) under high pressure up to $21 \mathrm{GPa}$, using plane wave-pseudopotential (PW-PP) approach in the framework of the density functional theory (DFT) and the density functional perturbation theory (DFPT) with the generalized gradient approximation (GGA) for the exchange-correlation functional. The results obtained are analyzed and compared with other data of the literature. The structural parameters and the piezoelectric coefficients calculated here agree well with other data of the literature. We found also that both the direct and converse piezoelectric coefficients increase with increasing pressure up to $21 \mathrm{GPa}$.
\end{abstract}

Keywords: Alp Semiconductor; Structural Parameters; Piezoelectric Coefficients; High Pressure.

\section{Introduction}

Over the last few years, investigation of several materials under high pressure and temperature has become an extremely important subject displaying explosive growth [1].

Due to its the remarkable physical properties such as high thermal conductivities, low dielectric, low densities and wide band-gap, the III-phosphides semiconductors have been receiving considerable attention, especially in its wide application towards electronic and optoelectronic devices [2].

Using first principles total-energy calculations based on the fullpotential augmented plane-wave plus local orbitals (FPLAPW+lo) method, Arbouche et al. [1] have investigated the structural phase transition under the effect of high pressure of some III-phosphide compounds (BP, GaP, AlP, InP). For the AlP compound, they found that this material transforms from the $\mathrm{B} 3$ to the B1 structure at a pressure of around $11.87 \mathrm{GPa}$.

Bouhemadou et al. [3] used the same approach to investigate the effect of the high pressure on the structural and mechanical properties of the same compounds. They found a linear dependence of the elastic constants versus the applied pressure. They estimated also the average sound velocity of the different materials and derived the Debye temperature from them.

Using first-principles calculations, Van Camp and Van Doren [4] studied some important physical properties of AlP in several phases. They found that AlP transforms to the metallic nickel-arsenide structure at a calculated pressure of around $8.3 \mathrm{GPa}$

In our previous work [5], the elastic constants, the piezoelectric coefficient and the electronic properties of AlP under high pressure were studied using ab-initio calculations in the framework of the density functional theory (DFT) and the density functional perturbation theory (DFPT) with the local density approximation (LDA). We found that the elastic constants and the piezoelectric coefficient increase monotonously with increasing pressure.

In other our recent work [6], the mechanical behavior and some other thermal properties of AlP were also systematically studied.
In the present contribution, we report on first-principles calculations of the structural parameters and the pressure effect on the piezoelectric coefficients of the cubic zinc-blende AlP material.

\section{Computational method}

The structural parameters and the piezoelectric coefficients calculated in the present study were carried out using ABINIT code [7] based on the pseudopotential plane-waves (PP-PW) approach in the framework of the DFT and the DFPT [8]. The TroullierMartins type pseudo-potentials which have been generated thanks to the FHI98PP code [9] were used to describe the interactions between the valence electrons and the nuclei and core electrons. The exchange-correlation energy was evaluated in the generalized gradient approximation (GGA) [10]. The total energy was found converged near the energy cutoff of $80 \mathrm{Ha}$. The Brillouin zone was sampled by $(6 \times 6 \times 6)$ Monkhorst and Pack mesh of $\mathrm{k}$ points [11]. For more detail on the calculation of the structural parameters and the piezoelectric coefficients of materials, please see for example our previous works $[5,6]$.

\section{Results and discussion}

\subsection{Normalized structural parameters}

Usually, the normalized structural parameters at fixed values of applied hydrostatic pressure were used to study the equation of state (EOS) of any compressed material. Both the normalized lattice parameter $\left(\mathrm{a}_{\mathrm{p}} / \mathrm{a}_{0}\right)$ and volume $\left(\mathrm{V}_{\mathrm{p}} / \mathrm{V}_{0}\right)$ versus pressure in the range from 0 to $21 \mathrm{GPa}$ for cubic zincblende AlP material were plotted in Fig. 1. From figure 1, we can observe that compression leads to decrease both $\left(\mathrm{a}_{\mathrm{p}} / \mathrm{a}_{0}\right)$ and $\left(\mathrm{V}_{\mathrm{p}} / \mathrm{V}_{0}\right)$ of AlP material. This is consequence of the diminishing in the nearest-neighbor distance of our material of interest. 


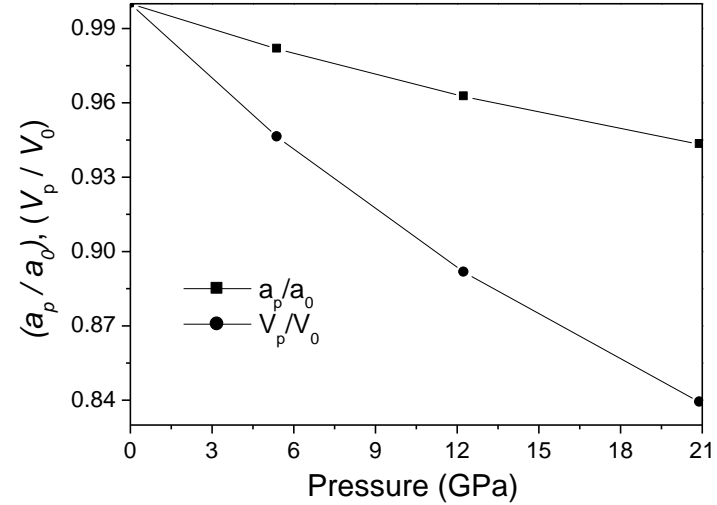

Fig. 1: Normalized Lattice Parameter $\left(A_{p} / A_{0}\right)$ and Volume $\left(V_{p} / V_{0}\right)$ Versus Pressure Up To $21 \mathrm{Gpa}$.

\subsection{EOS parameters}

In addition to Energy - Volume data; the equilibrium lattice constant a0, the bulk modulus $\mathrm{B}_{0}$, and its pressure derivatives $\mathrm{B}_{0}{ }^{\prime}$ can be determined also through the well-known pressure-normalized volume $\left(\mathrm{P}-\left(\mathrm{V} / \mathrm{V}_{0}\right)\right)$ data using the third-order Vinet EOS, which is given as follow $[12,13]$ :

$P(V)=3 B_{0}\left[\frac{1-\left(V / V_{0}\right)^{1 / 3}}{\left(V / V_{0}\right)^{2 / 3}}\right] \exp \left\{\frac{3}{2}\left(B_{0}{ }^{\prime}-1\right)\left[1-\left(V / V_{O}\right)^{1 / 3}\right]\right\}$

Where: $\mathrm{V}$ is the volume at pressure $\mathrm{P}$ and $\mathrm{V}_{0}$ is the volume at zeropressure, respectively.

The calculated $\left(\mathrm{P}-\left(\mathrm{V} / \mathrm{V}_{0}\right)\right)$ data in the range of pressure $(0$ to 21 $\mathrm{GPa}$ ) of AlP semiconductor was plotted in Fig. 2, along with the results reported Bouhemadou et al. [3].

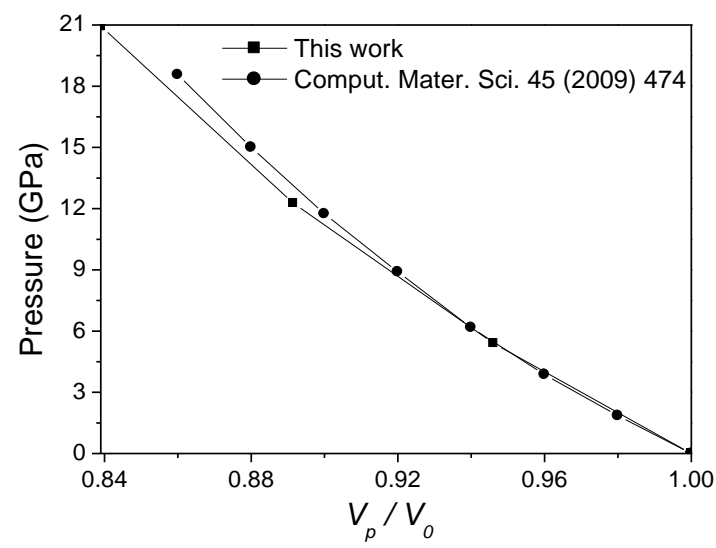

Fig. 2: Pressure Versus $\left(\mathrm{V}_{\mathrm{p}} / \mathrm{V}_{0}\right)$ of AlP Semiconductor.

Our calculated values of $\mathrm{a}_{0}, \mathrm{~B}_{0}$, and $\mathrm{B}_{0}{ }^{\prime}$ are: $5.498 \AA$, $87 \mathrm{GPa}$ and 3.65 , respectively. They are summarized in Table 1 and compared with other theoretical results [1-5].

Table 1: Equilibrium Lattice Constant $\mathrm{a}_{0}$, Bulk Modulus $\mathrm{B}_{0}$, and Its Pressure Derivatives $\mathrm{B}_{0}{ }^{\prime}$ of AlP, Compared to Other Theoretical Data [1-5]

\begin{tabular}{llll}
\hline Parameter & $\mathrm{a}_{0}(\AA)$ & $\mathrm{B}_{0}(\mathrm{GPa})$ & $\mathrm{B}_{0}{ }^{\prime}$ \\
\hline This work & 5.498 & 87 & 3.65 \\
Ref. [1] & 5.51 & 83.23 & 4.02 \\
Ref. [2] & 5.491 & 82 & - \\
Ref. [3] & 5.5163 & 82.46 & 4.16 \\
Ref. [4] & 5.461 & 87.5 & 3.8 \\
Ref. [5] & 5.436 & 86.29 & 4.05 \\
\hline
\end{tabular}

From the Table 1, it is observed that ao is in good agreement compared to other theoretical ones [1-5]. Our value (5.498 $\AA$ ) underestimate the theoretical one $(5.51 \AA)$ obtained from FP-LAPW+lo method [1] within only $0.22 \%$. Our obtained values of $\mathrm{B}_{0}$, and $\mathrm{B}_{0}{ }^{\prime}$ are also in good agreement compared to available theoretical ones [1-5]. Our obtained value $(87 \mathrm{GPa})$ of $\mathrm{B}_{0}$ underestimate the theo- retical one (87.5 GPa) obtained by Van Camp and Van Doren [4] within only $0.57 \%$.

\subsection{Crystal density}

The density g of material was related to the structural and the composition of its constituents as follow [5], [14], [15]

$$
g=\frac{\text { Z.M.u }}{a \cdot b \cdot c \sqrt{\sin ^{2} \alpha+\sin ^{2} \beta+\sin ^{2} \delta-2(1-\cos \alpha \cdot \cos \beta \cdot \cos \delta)}}
$$

Where: $\mathrm{Z}$ is the number of formula units in unit cell, $\mathrm{M}$ is the molecular weight of a formula unit, $\mathrm{u}$ is weight of an amu, $\mathrm{a}, \mathrm{b}$ and $\mathrm{c}$ are unit cell axes lengths, and $\alpha, \beta$ and $\delta$ are unit cell axes angles. The calculated crystal density at different values of pressure is plotted in Fig. 3. An analytical relation for the pressure dependence of the crystal density is given by the following a quadratic fit:

$\mathrm{g}=2.316+2.548 \times 10^{-2} \mathrm{p}-2.034 \times 10^{-4} \mathrm{p}^{2}$

Where $\mathrm{p}$ is given in $\mathrm{GPa}$, and $\mathrm{g}$ in $\mathrm{g} / \mathrm{cm}^{3}$.

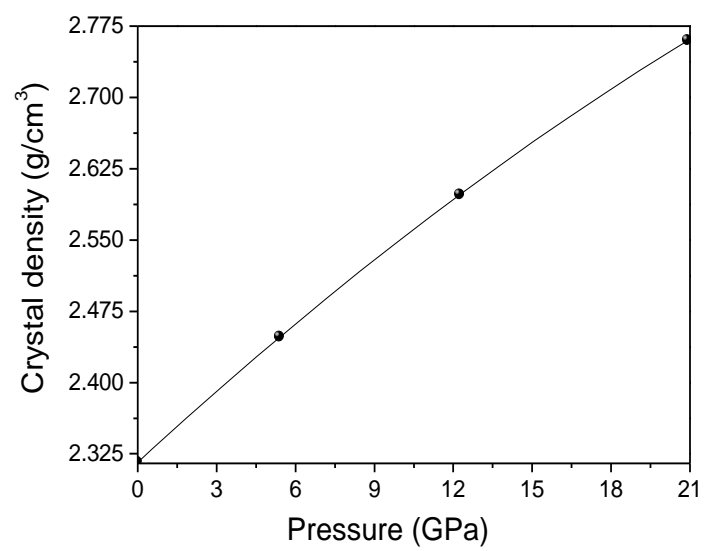

Fig. 3: Crystal density Versus Pressure of AlP up to $21 \mathrm{GPa}$.

At zero-pressure, our calculated value of the crystal density g was found at around $2.316 \mathrm{~g} / \mathrm{cm}^{3}$. Almost it is slightly lower than our previous value $\left(2.397 \mathrm{~g} / \mathrm{cm}^{3}\right)$ obtained from the local density approximation (LDA) [5], it is in general in agreement compared to the theoretical one $\left(2.355 \mathrm{~g} / \mathrm{cm}^{3}\right)$ reported by Ma et al. [2]

\subsection{Piezoelectric coefficients}

In the following section, we will discuss the piezoelectric properties of our material. Among the 5 symmetry classes belonging to the cubic system, only $\overline{4} 3 \mathrm{~m}$ and 23 classes exhibit the piezoelectric effect, for the 3 others classes, the piezoelectric effect being absent [5]. In the first case, the piezoelectric tensor contains only one constant called $\mathrm{e}_{14}$, it is usually expressed in $\mathrm{C} / \mathrm{m}^{2}[5],[16$, [17]:

$\underline{e}=\left(\begin{array}{cccccc}0 & 0 & 0 & e_{14} & 0 & 0 \\ 0 & 0 & 0 & 0 & e_{14} & 0 \\ 0 & 0 & 0 & 0 & 0 & e_{14}\end{array}\right)$

Figure 4 shows the dependence of $\mathrm{e}_{14}$ as a function of the pressure, along with the results reported in our previous work [5]. From figure 4 , we can observe that $\mathrm{e}_{14}$ increases monotonously with increasing pressure. This behavior of $\mathrm{e}_{14}$ was also observed in our previous work for AlP material [5] and for TIP compound [16]. At zero pressure, our value of $\mathrm{e}_{14}$ is around $-0.017 \mathrm{C} / \mathrm{m}^{2}$, it is slightly lower than the value $\left(0.051 \mathrm{C} / \mathrm{m}^{2}\right)$ obtained from the LDA [5], it is in general in agreement compared to the result $\left(-0.06 \mathrm{C} / \mathrm{m}^{2}\right)$ reported by Adachi [18]. An analytical relation for the pressure dependence of $\mathrm{e}_{14}$ can be given by the following a quadratic fit: 


$$
\mathrm{e}_{14}\left(\mathrm{C} / \mathrm{m}^{2}\right)=-0.0168+2.596 \times 10^{-2} \mathrm{p}-3.239 \times 10^{-4} \mathrm{p}^{2}
$$

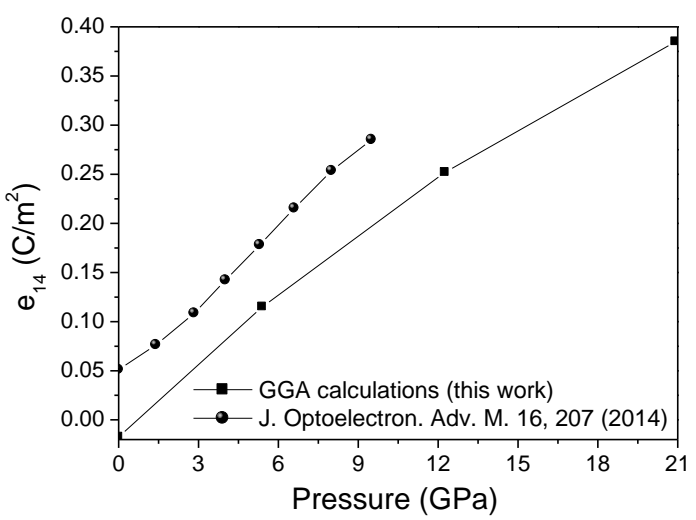

Fig. 4: Variations of $\mathrm{e}_{14}$ with Pressures of AlP, Along with the Theoretical One Reported in Our Previous Work [5].

In the converse piezoelectric effect, the piezoelectric tensor contains has also only one constant called $\mathrm{d}_{14}$, which is expressed in $10^{-12} \mathrm{~m} / \mathrm{V}$, or in $\mathrm{pC} / \mathrm{N}[16,17]$

Figure 5 shows the dependence of the converse piezoelectric coefficient $d_{14}$ as a function of the pressure, along with the theoretical one reported in our previous work [6]. From figure 5, we can observe that $\mathrm{d}_{14}$ increases also monotonously with increasing pressure. This behavior under pressure of $\mathrm{d}_{14}$ was also observed in our previous work for AlP material [6], and for TIP compound [17].

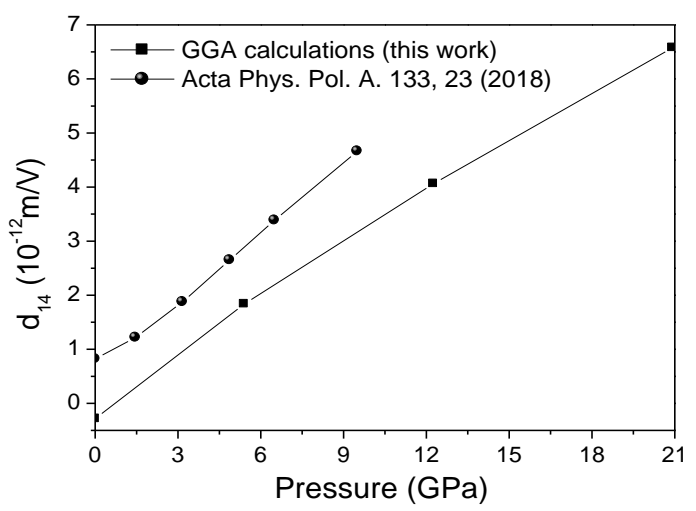

Fig. 5: Variations of $d_{14}$ with Pressures of AlP, Along with the Theoretical One Reported in Our Previous Work [6].

At zero pressure, our value of $\mathrm{d}_{14}$ is around $-0.282 \times 10^{-12} \mathrm{~m} / \mathrm{V}$, it is also slightly lower than our previous value $\left(0.823 \times 10^{-12} \mathrm{~m} / \mathrm{V}\right)$ obtained from the LDA [6]. An analytical relation for the pressure dependence of $\mathrm{d}_{14}$ can be given by the following a quadratic fit:

$$
\mathrm{d}_{14}(\mathrm{~m} / \mathrm{V})=-0.261+3.978 \times 10^{-1} \mathrm{p}-3.41 \times 10^{-3} \mathrm{p}^{2}
$$

\section{Conclusion}

Using the DFT and the DFPT with the GGA for the exchangecorrelation functional, we determine the equilibrium lattice constant, the bulk modulus, and its pressure derivatives of AlP material with cubic zinc-blende structure. The values obtained are in good agreement compared to other results of the literature. The pressure dependence of the direct and converse, piezoelectric coefficients up to $21 \mathrm{GPa}$ are also investigated, and compared to our previous results [5], [6] obtained with the LDA.

It was found that both the direct and converse, piezoelectric coefficients increase monotonously with increasing pressure.

To the best of our knowledge, there are no other data (except our previous works [5], [6]) available in the literature on the effect of pressure on $\mathrm{e}_{14}$ and $\mathrm{d}_{14}$ for this compound. Future other works will contribute with more tests for the validating of our results.

\section{References}

[1] O. Arbouche, B. Belgoumène, B. Soudini, Y. Azzaz, H. Bendaoud, and K. Amara, " First-principles study on structural properties and phase stability of III-phosphide (BP, GaP, AlP and InP) ", Computational Materials Science, Vol. 47, No. 3, (2010), pp. 685-692. https://doi.org/10.1016/j.commatsci.2009.10.009.

[2] H. Ma, J. Zhang, B. Zhao, Q. Wei, and Y. Yang, " First-principles study on mechanical and elastic properties of $\mathrm{B}_{\mathrm{x}} \mathrm{Al}_{1-\mathrm{x}} \mathrm{P}$ alloys ", AIP Advances, Vol. 7, No. 6, (2017), pp. 065007 (12 pages). https://doi.org/10.1063/1.4985254.

[3] A. Bouhemadou, R. Khenata, M. Kharoubi, T. Seddik, Ali H. Reshak, and Y. Al-Douri, " FP-APW + lo calculations of the elastic properties in zinc-blende III-P compounds under pressure effects ", Computational Materials Science, Vol. 45, No. 2, (2009), pp. 474479. https://doi.org/10.1016/j.commatsci.2008.11.013.

[4] P.E.Van Camp and V.E.Van Doren, "High pressure phase transitions in aluminum phosphide", Solid State Communications, Vol. 95, No. 3, (1995), pp. 173-175. https://doi.org/10.1016/00381098(95)00246-4.

[5] S. Daoud, N. Bioud, and N. Lebga, "Structural, elastic, piezoelectric and electronic properties of (B3) AlP compound under pressure", Journal of Optoelectronics and Advanced Materials, Vol. 16, No. (1-2), (2014), pp. 207- 214. https://joam.inoe.ro/index.php?option= magazine \&op=view\&idu $=3416 \&$ catid $=82$.

[6] S. Daoud, "Mechanical behavior of aluminum phosphide under pressure ", Acta Physica Polonica A, Vol. 133, No. 1, (2018), pp. 23-27. http://przyrbwn.icm.edu.pl/APP/PDF/133/app133z1p06.pdf. https://doi.org/10.12693/APhysPolA.133.23.

[7] The ABINIT code is a common project of the Université Catholique de Louvain, Corning Incorporated, and other contributors. http://www.abinit.org.

[8] E. Engel, and R.M. Dreizler, Density functional theory, SpringerVerlag Berlin Heidelberg, New York, (2011). ISBN 978-3-64214090-7. https://doi.org/10.1007/978-3-642-14090-7.

[9] M. Fuchs and M. Scheffler, "Ab initio pseudopotentials for electronic structure calculations of poly-atomic systems using densityfunctional theory", Computer Physics Communications, Vol.119, No. 1, (1999), pp. 67-98. https://doi.org/10.1016/S00104655(98)00201-X.

[10] J.P. Perdew, K. Burke, and M. Ernzerhof, "Generalized gradient approximation made simple", Physical Review Letters, Vol. 77, No. 18, (1996), $\quad$ pp. $3865 \quad$ - 3868. https://journals.aps.org/prl/abstract/10.1103/PhysRevLett.77.3865. https://doi.org/10.1103/PhysRevLett.77.3865.

[11] H. J. Monkhorst, and J. D. Pack, "Special points for Brillouin-zone integrations", Physical Review B, Vol. 13, No. 12, (1976), pp. 5188

https://doi.org/10.1103/PhysRevB.13.5188.

[12] A. R. Oganov, J.P. Brodholt, and G.D. Price, Ab initio theory of thermoelasticity and phase transitions in minerals. EMU Notes in Mineralogy Vol. 4, Chapter 5 ('Energy Modeling in Minerals', edited by C.M. Gramaccioli), (2002). pp.83-170.

[13] S. Daoud, R. Mezouar and A. Benmakhlouf, " Equation of state and some other properties of rock-salt AlN ", International Journal of Physical Research, Vol. 6, No. 1, (2018), pp. 49-52. https://doi.org/10.14419/ijpr.v6i1.10754.

[14] S. Daoud and N. Bioud, " Structural properties of (B3) TIP under pressure", International Journal of Physical Research, Vol. 2, No. 2, (2014), pp. 50-55. https://doi.org/10.14419/ijpr.v2i2.3100.

[15] S. Daoud and N. Lebga, " Structural and electronic properties of boron- bismuth compound under pressure ", International Journal of Physical Research, Vol. 4, No. 1, (2016), pp. 1-5. https://doi.org/10.14419/ijpr.v4i1.5753.

[16] S. Daoud, N. Bioud, and N. Lebga, "Mechanical, piezoelectric and some thermal properties of (B3) BP under pressure", Journal of Central South University, Vol. 21, No. 1, (2014), pp. 58-64. https://doi.org/10.1007/s11771-014-2394-4.

[17] S. Daoud, "Mechanical and piezoelectric properties, sound velocity and Debye temperature of thallium-phosphide under pressure", International Journal of Advanced Research in Physical Science, Vol. 1, No. 6, (2014), $1-11$. www.arcjournals.org/pdfs/ijarps/v1i6/1.pdf.

[18] S. Adachi, "Properties of group-IV, III-V and II-VI semiconductors", John Wiley \& Sons, England, (2005). ISBN 0-470-09032-4. 